\title{
New MRI-safe Implant Electrode Design
}

\author{
Steven O. McCabe and Jonathan B. Scott \\ Department of Engineering, The University of Waikato, Hamilton, New Zealand
}

\begin{abstract}
Medical implants often prevent patients having Magnetic Resonance Imaging (MRI) scans because the leads behave as antennas with respect to the RF excitation and cause hazardous heating in neural tissue. This manuscript describes an approach that virtually eliminates the risk of RF heating by means of easily-incorporated, mutually-coupled filars. The resulting leads need be neither physically larger nor significantly more costly than existing designs. Combined with thin insulation and surface roughening techniques, this manuscript represents the first complete release of recently-patented technologies. Both simulations and measurements at $128 \mathrm{MHz}$ are presented to confirm performance in 3-Tesla MRI machines.
\end{abstract}

\section{INTRODUCTION}

The RF hazard that accompanies MRI scanners for patients whose bodies contain conductive leads is well known. [1] The MRISAFETY.COM web site is used by radiographers the world over to check if an implant is safe or conditionally safe before a patient is scanned. [2] Despite numerous patent filings, only one product claiming to be MRI-safe has appeared, and that product addresses only $64 \mathrm{MHz}, 1.5 \mathrm{~T}$ machines. [3], [4]

\section{THIS WORK}

This manuscript introduces the "decoy" technique. When combined with existing thin-insulation techniques [3] and surface treatments [4]-[6], it is expected to achieve unconditional MRI-safe performance. Decoy requires as little as a single extra filar to be incorporated into an implant lead. It operates through mutual coupling to an added filar of selected length and with thin insulation. [3], [5], [7] The approach will be discussed in detail in section IV.

\section{ORDINARY IMPLANT LEAD}

The distal electrodes at the end of a typical implant lead for SCS is shown in Fig. 1. Such leads can range up to several hundred millimeters in length, or 1-2 wavelengths in vivo. [8] When an implanted electrode appears to be just short of half a wavelength, concentrated current around the tip leads to tissue heating. [3] Fig. 2 shows agreement between heating predicted by simulation, measured with a torso phantom in a commercial $3 \mathrm{~T}$ MRI machine with a whole-body SAR of $1 \mathrm{~W} / \mathrm{kg}$ as shown in Fig. 3, and measured with $128 \mathrm{MHz}$ dipole radiators and a CW power amplifier. [9] The safety threshold of $1-2{ }^{\circ} \mathrm{C}$ as endorsed by the International Commission on Non-Ionizing Radiation Protection (ICNIRP) [10], is exceeded by more than ten times at the worst-case length.

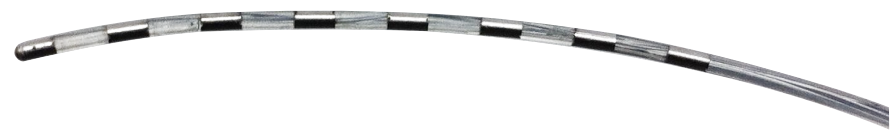

Fig. 1. Distal end of an "Octrode" lead intended for SCS. Eight platinum electrodes, each $3 \mathrm{~mm}$ long and $1.3 \mathrm{~mm}$ in diameter, are separated by $4 \mathrm{~mm}$ insulating spacers.

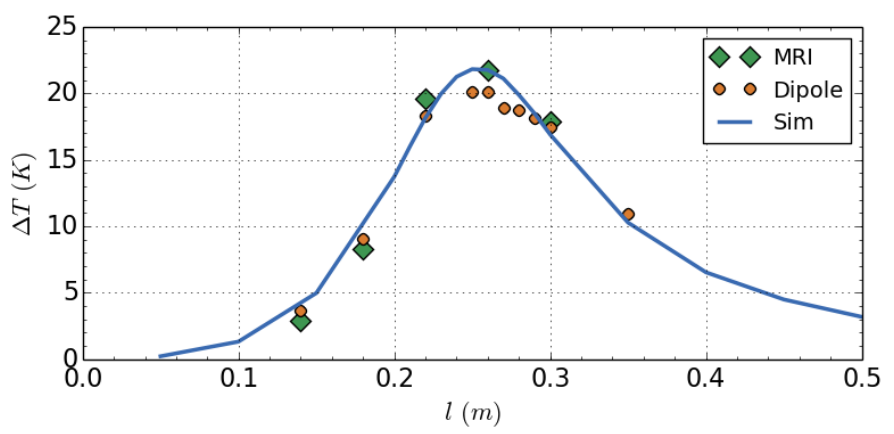

Fig. 2. Simulated and measured temperature rise $\Delta T$ near the distal electrode of an insulated wire after 5 minutes in a 3T MRI machine. Measurements from the dipole test method yield a similar profile.

\section{Lead With Mutually-Coupled Decoy}

Near-field interaction is essential in the operation of antenna arrays. A Yagi-Uda antenna for example, employs directive and reflective elements mutually coupled to a driven dipole, forming a highly directional antenna. [11] Implant leads may be regarded as "Antennas in matter", a subject that has been dealt with in detail by King. [12] In the case of an implant electrode, an additional and preferably bare conductor, hereinafter referred to as decoy, can be attached to the exterior of the lead. The direct contact between the decoy and tissue provides a damping effect in accordance with results reported in the literature. [3], [6] This damping is "felt" by the lead conductors through mutual coupling. Currents induced in the lead conductors by MRI excitation are reduced, leading to abated joule heating at the electrode-to-tissue interfaces.

To confirm this expectation, simulations were performed in COMSOL Multiphysics 4.4 with a phantom model set according to ASTM F2182-11a [13]. We used the same

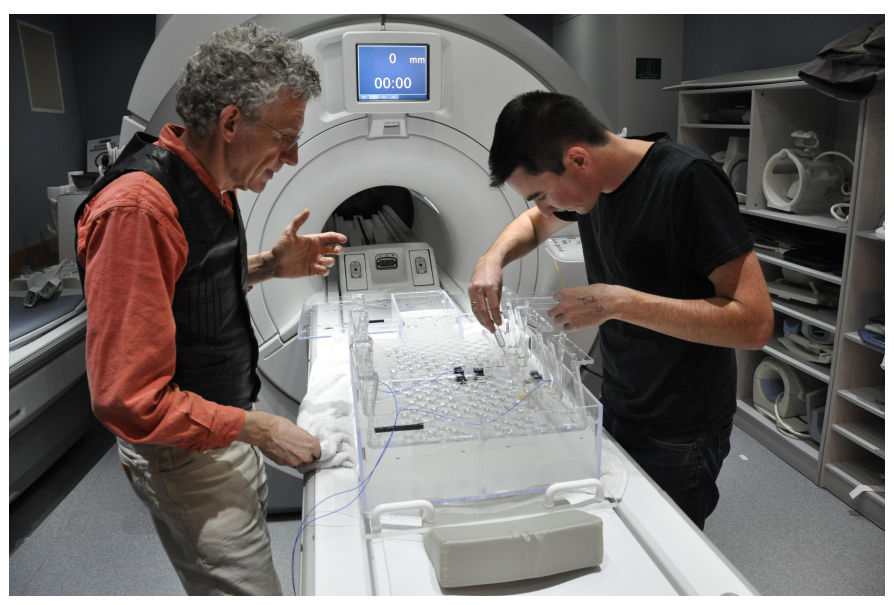

Fig. 3. Clear acrylic phantom on the bed of the MRI machine with operators. 


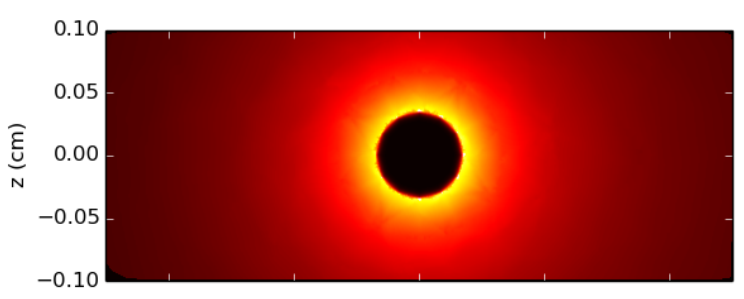

(a)

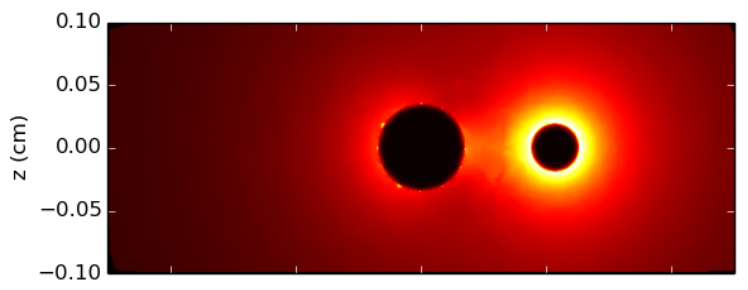

(b)

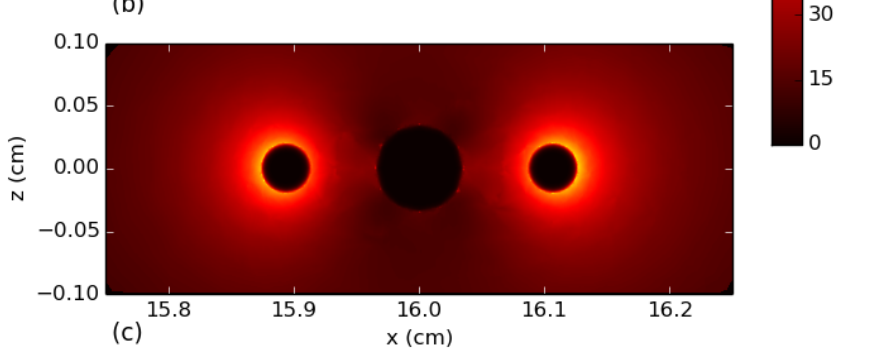

Fig. 4. Midpoint cross-section showing the simulated magnitude of the $\mathrm{H}$ field surrounding an (a) ordinary electrode lead (b) electrode lead with single bare $0.6 l$ decoy (rightmost conductor) (c) electrode lead with two bare $0.9 l$ decoys (outermost conductors). The length of the electrode lead is $25 \mathrm{~cm}$.

simulation arrangements that have been previously reported in [3]. Referring to Fig. 4, simulation predicts that the current induced in a single conductor insulated from the surrounding saline medium after the fashion of an implant lead filar (a) will be significantly reduced when that filar is accompanied by a second, uninsulated filar (b), and even more so in the case of two mutually-coupled filars (c).

Fig. 5(a) shows simulated predictions of the distal heating for an assortment of leads with length $l$, each having a single decoy of specified proportionate length. A decoy of length $0.6 l-0.7 l$ seems to produce the least amount of heating over the range. Heating is virtually eliminated for leads with two decoys as is shown Fig. 5(b), when the length of each equivalent decoy is between $0.7 l-0.9 l$.

\section{Measured Results}

Fig. 6 depicts test leads with diameters and insulation thickness identical to ones that have been previously reported in the literature [5], [9], but to which we fitted adjacent, bare, "decoy" filars. These are used to verify simulations against measurements in MRI and in the lab.

The measured heating of leads with a single $0.6 \mathrm{l}$ decoy and a $0.9 l$ decoy are shown in Figs. 7(a) and 7(b), respectively. Previously simulated results are overlaid in the same figures. For the lead with the $0.6 l$ decoy, we measure a temperature rise of less than $6^{\circ} \mathrm{C}$ over the entire lead length range. The $0.9 l$ decoy produces an even smaller temperature rise of just $2{ }^{\circ} \mathrm{C}$ when the lead length is short.
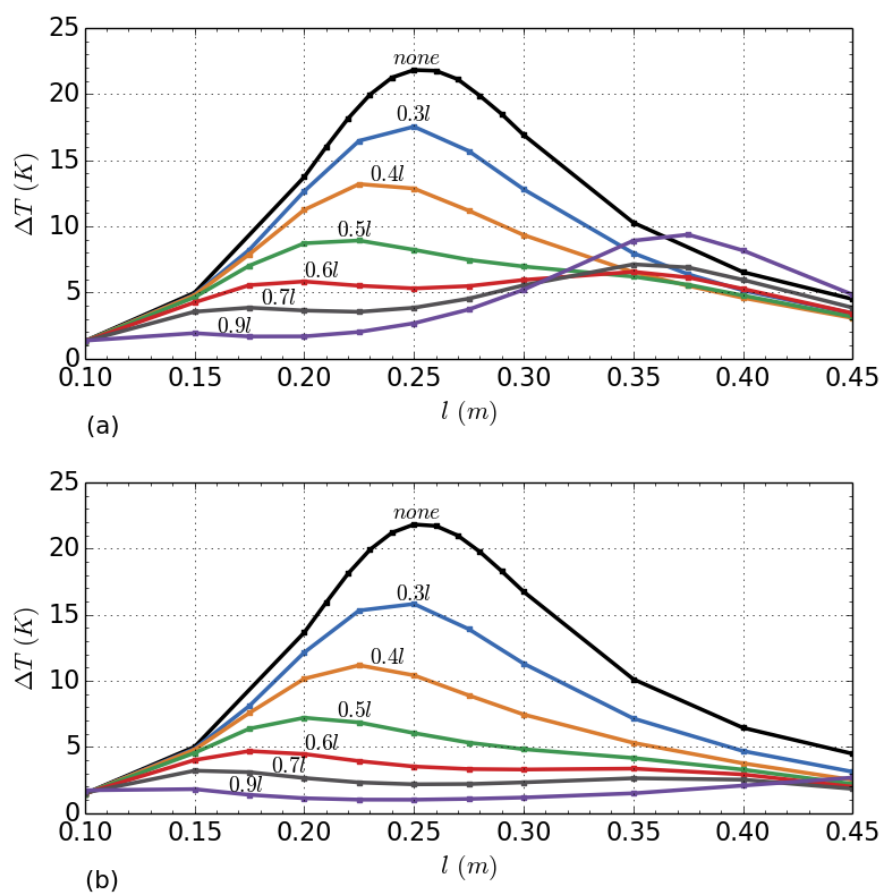

Fig. 5. Simulated heating profile $(t=5 \mathrm{~min}, \mathrm{SAR}=1 \mathrm{~W} / \mathrm{kg}$ ) at the distal electrode as a function of lead length, for a range of electrode leads each with (a) a single decoy (b) two decoys. Electrode leads differ by a fixed decoyto-lead length ratio in the range of $0.3-0.9 l$. An ordinary electrode lead without a decoy has the profile denoted by "none".

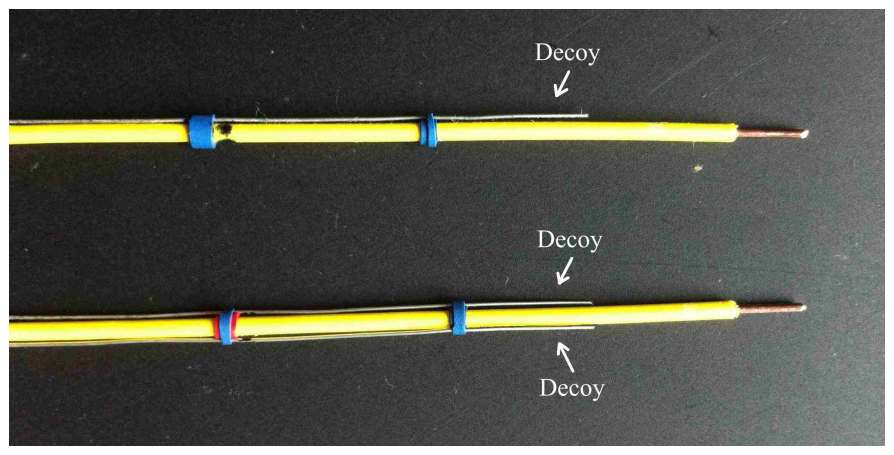

Fig. 6. The single decoy (top) and dual decoy (bottom) test leads comprise of $800 \mu \mathrm{m}$ dia. copper wire coated with $350 \mu \mathrm{m}$ worth of insulation covering all but $6 \mathrm{~mm}$ from one end (the electrode). $400 \mu \mathrm{m}$ dia. bare copper wire adhered alongside the lead forms the decoy(s).

Fig. 8 shows a further reduction in heating when the same leads have an additional and identical decoy. A rise of just $0.5^{\circ} \mathrm{C}$ is measured when the lead is $30 \mathrm{~cm}$ long with $0.9 \mathrm{l}$ decoys, corresponding to a $97 \%$ reduction when compared to the ordinary implant lead in Fig. 2.

\section{DISCUSSION}

A cost-effective implant electrode design that meets the ICNIRP standard for safe heating has been achieved through the addition of uninsulated filars of preselected length, to the exterior of the lead. Further improvement to the safety margin may be possible by increasing the ac resistance of the lead conductors to a few hundred ohms per meter. Roughening the surface of lead filars as described in [4] is expected to provide 


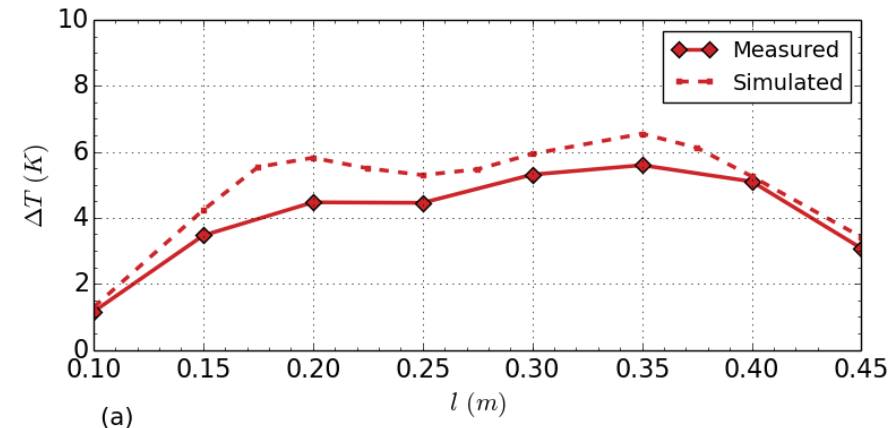

(a)

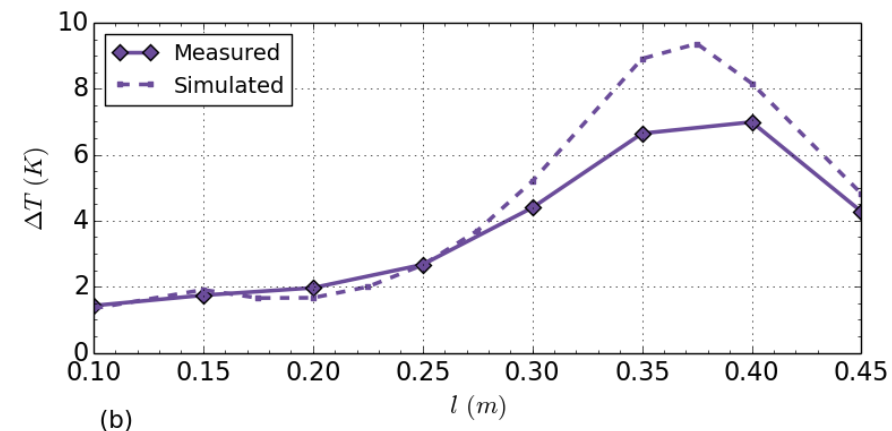

Fig. 7. Measured heating profile $(t=5 \mathrm{~min}, \mathrm{SAR}=1 \mathrm{~W} / \mathrm{kg}$ ) of electrode leads attached alongside single decoys with decoy-to-lead length ratios of (a) $0.6 l$ and (b) $0.9 l$.


Fig. 8. Measured heating profile ( $t=5 \mathrm{~min}, \mathrm{SAR}=1 \mathrm{~W} / \mathrm{kg}$ ) of electrode leads attached between two decoys with decoy-to-lead length ratios of (a) $0.6 l$ and (b) $0.9 l$. that increased RF impedance without negatively impacting on implant battery life implied by dc resistance. [3], [6]

\section{CONCLUSION}

We introduced a novel technique that will prevent hazardous heating normally mediated by implanted conductors in 3-Tesla MRI machines. The technique relies upon the use of mutual coupling applied simultaneously with techniques that the authors have previously reported elsewhere. Simulation and measurement in a phantom confirm the impact of the technique. Two patents have been filed so far.

\section{ACKNOWLEDGEMENT}

The authors wish to thank Stephen Butler of Midland MRI for his kind assistance.

\section{REFERENCES}

[1] J. A. Nyenhuis, P. Sung-Min, R. Kamondetdacha, A. Amjad, F. G. Shellock and A. R. Rezai "MRI and Implanted Medical Devices: Basic Interactions With an Emphasis on Heating", IEEE Transactions on Device and Materials Reliability, vol. 5, no. 3, pp. 467-480, Sept. 2005.

[2] "MRISAFETY.COM, Your Information Resource for MRI Safety, Bioeffects, \& Patient Management", http://www.mrisafety.com/ retrieved Sept 2014.

[3] S. McCabe and J. Scott, "Cause and amelioration of MRI-induced heating through medical implant lead wires," Proceedings of the 21st Electronics New Zealand Conference, Hamilton, 20-21 Nov. 2014, pp. $34-40$.

[4] J. B. Scott and S. O. McCabe, "Implant conductor with improved radio frequency properties", New Zealand provisional patent 708633, Filed on May 29, 2015.

[5] S. O. McCabe, "MRI-safe Implantable Electrode Leads", PhD thesis, The University of Waikato, 2016.

[6] S. McCabe and J. Scott, "Electromagnetic techniques to minimize the risk of hazardous local heating around medical implant electrodes during MRI scanning," European Microwave Conference 2015, Paris, 7-10 Sept. 2015.

[7] S. O. McCabe and J. B. Scott, "Implant conductor assembly with improved radio frequency properties", New Zealand provisional patent 714212, Filed on Nov 16, 2015.

[8] St. Jude Medical, "Percutaneous Leads for SCS", http: //professional.sjm.com/products/neuro/scs/percutaneous-leads/ percutaneous-leads-for-scs, retrieved November 2015.

[9] S. McCabe and J. Scott, "Technique to assess the compatibility of medical implants to the RF field in MRI," Asia-Pacific Microwave Conference 2015. Nanjing, 6-9 Dec. 2015.

[10] "ICNIRP Statement on: Medical Magnetic Resonance (MR) Procedures: Protection of Patients", International Commission on Non-Ionizing Radiation Protection (ICNIRP), Health Phys., vol. 87 no. 2, pp. 197216, Aug. 2004.

[11] S. Ramo, J. R. Whinnery and T. Van Duzer, Fields and Waves in Communication Electronics, Second Edition, John Wiley \& Sons, Inc, pp. 147-150, 1984.

[12] R. W. P. King and G. S. Smith, "Antennas in matter: fundamentals, theory, and applications", The MIT Press, Cambridge, MA, 1981.

[13] F2182-11a Standard Test Method for Measurement of Radio Frequency Induced Heating On or Near Passive Implants During Magnetic Resonance Imaging, ASTM International, West Conshohocken, PA, USA, 2010. 